\title{
Surgical Removal of Embolized PDA Device from Descending Thoracic Aorta - A Case Report
}

\author{
M Badrul Alam¹, Kazi Abul Hasan², M ZahiduL Islam², SA Quader², \\ M Sajedul Bari, AM Asif Rahim ${ }^{1}$ \\ ${ }^{1}$ Department of Cardiac Surgery, NICVD, ${ }^{2}$ Department of Pediatric Cardiac Surgery, NICVD
}

\begin{abstract}
:
Key Words:

Patent ductus

Closure of patent ductus arteriosus (PDA) with device or coil is presently the first line of therapy.but it has got different acute and late complications. A 3 years old male child presented with history of percutaneous closure of the PDA Amplatzer device 18 months back. Now he presented with arteriosus, Amplatzer device dislodgement of the device to descending thoracic aorta and reappearance of a large PDA. The child underwent surgical closure of PDA and removal of the device from descending thoracic aorta. We are presenting this case as one of the uncommon late complications of device closure of PDA.
\end{abstract}

(Cardiovasc. j. 2016; 8(2): 158-160)

\section{Introduction:}

Failure of normal physiologic closure of the Ductus arteriosus during the newborn period may lead to congestive heart failure and pulmonary insufficiency. Early surgical duct ligation is normally undertaken when pharmacologic therapy with indomethacin fails or is contraindicated. Percutaneous duct closure as an alternative to surgical therapy has become popular now. But there are some acute and late complications of PDA device closure. Dislodgement of device is one of them which demands urgent surgical removal of device.

\section{Case Report:}

A 1 year old male child with a patent ductus arteriosus with bidirectional blood flow, and severe pulmonary hypertension underwent percutaneous closure with a Amplatzer Duct Occlusion device 18 months back. After the procedure the mean pulmonary artery pressure decreased to $30 \mathrm{~mm}$ $\mathrm{Hg}$ with a good early outcome.

Echocardiography performed 18 months later demonstrated reappearance of patent ductus arteriosus with no evidence of device and persistently elevated pulmonary artery pressures. Fluoroscopy determined that the Amplatzer occlusion device had embolized into the descending thoracic aorta approximately $1 \mathrm{~cm}$ distal to the ductal opening (Fig 1).
Percutaneous attempts to recover the device were unsuccessful, and the patient was referred for surgical device removal. Chest was opened through a left posterolateral thoracotomy in the $4^{\text {th }}$ Intercostal space, a short patent ductus arteriosus was noted. The occlusion device was identified in

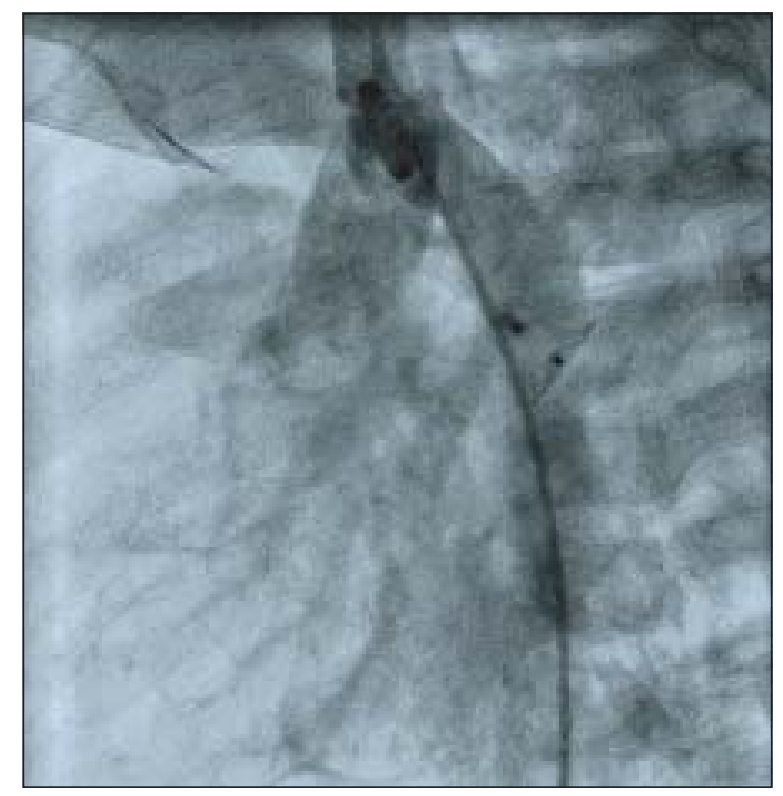

Fig.-1: Arteriogram of a patient 18months after transcatheter closureof patent ductus arteriosus with an Amplatzer Duct Occluder device.The occluder device embolized and became embedded in the intima of the descending thoracic aorta.

Address of correspondence: Dr. Md. Badrul Alam, Department of Cardiac Surgery, National Institute of Cardiovascular Diseases, Dhaka, Bangladesh.Email: alambadrul82@gmail.com 
the descending aorta by palpation. The ductus, the descending thoracic aorta and Pulmonary artery was dissected, After mobilizing vascular structures, Control was taken with tapes, Clamps were applied to the aortic isthmus, the descending aorta below the level of the device, and the pulmonary artery end of the ductus. The duct was closed with multiple ligature and Transfixation with $5 / 0$ prolene suture .

The descending aorta was opened by an incision over the Amplatzer device, aortotomy was extended both proximally and distally to provide exposure to the occlusion device. The large distal flange of the device had become embedded in the intimal layer of the aorta, and the device was tightly bonded to the aortic wall by a layer of pannus that had formed. The device was sharply dissected from the pannus. Portions of the pannus were also removed.

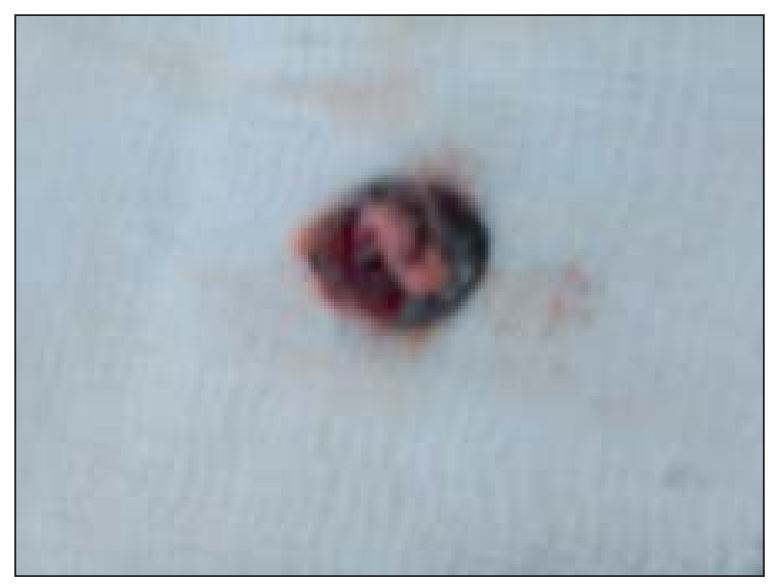

Fig.-2: The Amplatzer device and pannus attached on its surface, Just after surgical removal.

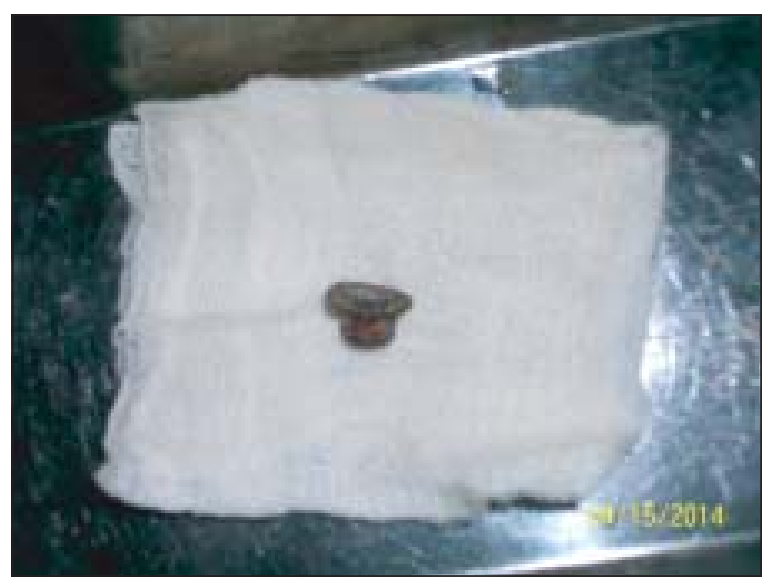

Fig.-3: The Amplatzer device after it was cleaned and washed with Normal saline.
The medial layer of the aorta was normal. The aortotomy was directly closed in two layers, without narrowing the lumen, and the mediastinal pleura was reapproximated,Chest was closed in layers, Keeping a chest drain insitu. The patient's postoperative course was uneventful with full recovery.

\section{Discussion:}

Percutaneous arterial duct closure was first reported by Porstmann in 1971 and is now widely applied. ${ }^{1}$ This case study underscores the importance of careful intermediate-term and long-term clinical follow-up of these patients. Although several studies demonstrate immediate and short-term safety and efficacy of percutaneous duct closure only a few reports describe long-term (5 years) clinical outcomes. ${ }^{2,3}$ When the utility of transcatheter arterial duct closure is evaluated, it is important to compare the results with those of surgical therapy. Since the first successful ligation of a patent ductus arteriosus in $1938,{ }^{4}$ countless patients have undergone successful surgical duct closure, most of them with decades of follow-up. In the current era, surgical duct ligation or division is highly effective, with essentially no associated operative mortality. ${ }^{5,6}$ Several retrospective studies comparing the results of surgical versus transcatheter arterial duct closure have suggested that surgical duct closure is more efficacious and generally less costly than transcatheter duct closure. ${ }^{6,7}$

\section{Conclusion:}

A clinical trial is needed to provide patients and their caregivers with the information necessary to give full and informed consent before surgical or catheter-based intervention. Especially in Bangladesh where cost of the procedure is a big issue, still surgical closure of PDA is more suitable, safe and cost effective. Failure of device closure lead to patient in life risk that require urgent surgery as a last attempt to remove device and ligation of PDA as well as to save the patient's valuable life.

\section{Conflict of Interest - None.}

\section{References:}

1. Porstmann W, Wierny L, Warnke H, Gerstberger G, Romaniuk PA. Catheter closure of patent ductus arteriosus. 62 cases treated without thoracotomy. Radiol Clin North Am 1971;9:203-218. 
2. Pass RH, Hijazi Z, Hsu DT, Lewis V, Hellenbrand WE.Multicenter USA Amplatzer patent ductus arteriosus occlusion device trial: initial and one-year results. J Am Coll Cardiol 2004;44:513-519.

3. Masura J, Tittel P, Gavora P, Podnar T. Long-term outcome of transcatheter patent ductus arteriosus closure using Amplatzer duct occluders. Am Heart J 2006;151:755 e7-10.

4. Gross RE, Hubbard JP. Landmark article Feb 25, 1939: surgicalligation of a patent ductus arteriosus. Report of first successful case. By Robert E. Gross and John P. Hubbard. JAMA 1984;251:1201-1202.

5. Mavroudis C, Backer CL, Gevitz M. Forty-six years of patient ductus arteriosus division at Children's Memorial
Hospital of Chicago. Standards for comparison. Ann Surg 1994;220:402-409.

6. Gray DT, Fyler DC, Walker AM, Weinstein MC, Chalmers TC. Clinical outcomes and costs of transcatheter as compared with surgical closure of patent ductus arteriosus. The patent ductus arteriosus closure comparative study group. $N$ Engl J Med 1993;329:1517-1523.

7. Hawkins JA, Minich LL, Tani LY, Sturtevant JE, Orsmond GS,McGough EC. Cost and efficacy of surgical ligation versus transcatheter coil occlusion of patent ductus arteriosus. J Thorac Cardiovasc Surg 1996;112:1634-1638. 